\title{
Clinical Events Associated with Acupuncture Intervention for the Treatment of Chronic Inflammation Associated Disorders
}

\author{
Hua Bai, ${ }^{1}$ Senlei Xu, ${ }^{1}$ Qiulan $W u,{ }^{2}$ Shanshan $X{ }^{3}{ }^{3}$ Ke Sun, ${ }^{1}$ Jiahong Wu, ${ }^{1}$ Xuefeng Xia, \\ Yuchen Liu, ${ }^{1}$ Hongru Zhang $\left({ }^{10},{ }^{1}\right.$ and Shengfeng $\mathrm{Lu}\left(\mathbb{1}^{1}{ }^{1}\right.$ \\ ${ }^{1}$ The Second Clinical Medical College, Nanjing University of Chinese Medicine, Nanjing, 210023 Jiangsu, China \\ ${ }^{2}$ Affiliated Hospital of Integrated Traditional Chinese and Western Medicine, Nanjing University of Chinese Medicine, Nanjing, \\ 210028 Jiangsu, China \\ ${ }^{3}$ Department of Acupuncture, Yuhua Community Health Service Center, Nanjing, 210012 Jiangsu, China
}

Correspondence should be addressed to Hongru Zhang; zhr5001@163.com and Shengfeng Lu; lushengfeng@njucm.edu.cn

Received 31 January 2020; Accepted 16 June 2020; Published 27 June 2020

Academic Editor: Alex Kleinjan

Copyright ( 92020 Hua Bai et al. This is an open access article distributed under the Creative Commons Attribution License, which permits unrestricted use, distribution, and reproduction in any medium, provided the original work is properly cited.

Acupuncture is a key component of Chinese medicine. It describes a series of procedures involving the stimulation of skin through penetration of fine, single-use, sterile needles that result in the release of neurotransmitters. Although its use is on the growing trend, considerable controversy surrounds its value as a therapy. Standard randomized controlled trials that adhere to the accepted criteria should be conducted in the future to ensure the effectiveness of acupuncture. This article summarizes the current evidence regarding the use of acupuncture. It includes a description of the history, mode of operation, treatment of a variety of chronic disorders related to inflammation, and future directions for acupuncture use. Published clinical trials support the view that acupuncture is a possible candidate for the treatment of several chronic inflammation-related disorders.

\section{Introduction}

The anti-inflammatory effect of acupuncture is a key analgesic mechanism of reducing pain [1]. Adverse effects associated with acupuncture are rare and minor [2]. Hence, it is a relatively safe complementary medicine to use when handled by qualified practitioners [3]. Although acupuncture in TCM has been used for the treatment of many disorders, in this review, we took a comprehensive literature survey to determine the clinical trials for its effectiveness in chronicinflammation associated diseases.

\section{History of Acupuncture Use}

Although acupuncture has been dated back to 3000 years in China, stones and bones aging to $6000 \mathrm{BC}$ have been identified with traces of acupuncture use [4]. Ranging from 1500 to $1700 \mathrm{AD}$, the Dutch East India Company initiated the concept of acupuncture into European medical practice. The first-ever clinical report on acupuncture was published as
"On Acupuncturation." in the year 1802 by the British physician William Coley. A series of then acupuncture related publications led to positive opinions about acupuncture among the medical community in Europe. The early 1800s saw the entry of acupuncture related publications into US medical journals. After a sequence of fluctuations, the National Institutes of Health (NIH), in 1997, acknowledged acupuncture for its benefits in the field of clinical medicine and recommended it to be included in the scheme of medical schools [5]. In 1979, the World Health Organization (WHO) organized a meeting which identified 43 different diseases that could be treated by acupuncture. It also published a book in 2003 to determine the clinical trials performed related to acupuncture [6].

\section{Acupuncture-Modus Operandi}

A loss of balance between the yin and yang energies is understood as a disease in Chinese medicine. Acupuncture relies on maintaining a balance between yin and yang through 
the use of several acupoints and 12 meridians. Among the 12 meridians, yin meridians include lung, spleen, heart, kidney, pericardium, and liver. Large intestines, stomach, small intestines, bladder, triple energizer, and gall-bladder are defined as yang meridians. The circulation of $Q i$, a vital life force that flows through the body is supposedly regulated by acupuncture via 12 meridians in a discrete order as mentioned in Chinese medicine body clock [7]. Acupuncture stimulation through the insertion of fine, single-use, sterile needles can result in the release of neurotransmitters such as endorphins, serotonin, enkephalins, gammaaminobutyric acid, norepinephrine, and dopamine [8]. Appropriate use of combinations in acupuncture can result in proper flow of energy through the meridians [9]. The effects of acupuncture are comparable to the effects observed after the use of capsaicin, a bioactive component of chili pepper [10].

\section{Therapeutic Potential of Acupuncture in Chronic Inflammation Related Disorders}

Acupuncture is effective as first-line or adjunct medication for treatment of chronic inflammation associated disorders such as allergic rhinitis, anxiety, asthma, atherosclerosis, and associated myocardial infarction, cancer, rheumatoid arthritis, stress, and Crohn's disease. The intervention is considered to be systemically effective and safe in the management of diseases associated with various regions of the human body [11-19]. Local-distal combination is widely used for intervention using acupuncture, whereas, local administration is effective for a local symptom. Further research on the comparison of effectiveness is warranted [20]. Local-distal acupoints such as Bladder 40 had been identified for specific disorders. These acupoints were found to be effective for the treatment of inflammationassociated disorders in comparison with local administration [21-25]. Dr. Tan's strategy applies a systemic approach to treat a patient. It is a novel approach to manage or cause significant alleviation of several disorders through the use of 12 points $[26,27]$.

4.1. Allergic Rhinitis. Allergic rhinitis is the most common respiratory disease. Acupuncture is a nasal obstruction and sneezing of 61 persistent allergic rhinitis patients improved at week 2 of acupuncture treatment at the 5th Affiliated Hospital of Sun Yat-Sen University, China. The sphenopalatine acupoint located in the cheek near Xiaguan was used for stimulation. The total nasal symptom score was significantly lower compared to the control [28]. Twenty-six patients aging 18 to 70 were selected to study the effects of acupuncture on seasonal allergic rhinitis at the RMIT University, Victoria, Australia. Ying Xiang, Yintang, and Fengchi were the acupoints used for stimulation. A significant improvement was observed in sneezing, watery rhinorrhoea, nasal congestion, itching nose (symptoms associated with nose), itching eyes, and eye-watering (nonnasal symptoms) [29].

A nonrandomized controlled design was used at Zhejiang Hospital, Hangzhou, China, to evaluate the effect and safety of acupuncture therapy on participants with moderate to severe allergic rhinitis. The thirty-six patients treated with acupuncture recovered effectively in equivalence to the effects of oral cetirizine hydrochloride tablets after four weeks of treatment. The acupoints used were Ying Xiang, Shang Xing, Fengchi, Quchi, Xue Hai, Fei Shu, Ge Shu, and Pishu. The scores for sneezing, runny nose, stuffy nose, nasal itching, and turbinate edema were similar among both the groups [30]. A multicenter, randomized, parallel-controlled study was conducted based at two centers in South Korea and one in China. The acupoints used were bilateral $\mathrm{He} \mathrm{Gu}$, Ying Xiang, Sibai, Zu San Li, unilateral Yintang, and Shang Xing. The total nasal and nonnasal symptom score of 97 patients treated with acupuncture was reduced in comparison to control groups after 4 weeks of treatment [31]. All these studies indicate that allergic rhinitis could be treated effectively by using acupuncture.

4.2. Asthma. Asthma is considered to be one among the widespread chronic respiratory diseases [32]. This childhood disorder causes limitations in the airflow of the airways in the 300 million affected individuals around the world and kills an estimate 346,000 every year [14]. Acupuncture has a long tradition in the treatment of asthma [33]. In an interesting study conducted at Jinan Hospital of TCM, China, acupuncture at Yuji meridian relieved the acute attack of bronchial asthma in two hundred and eighty-nine cases. The effects of 30minute needle-retaining were comparable to the effects of $200 \mu \mathrm{g}$ salbutamol aerosol [34]. Improved pulmonary function and reduced asthma attack were observed in ninety acute stage asthma cases at the Tianjin City Hospital of TCM, China, after acupuncture treatment [35]. Acupuncture significantly improved the symptoms of asthma and the Visual Analogue Scale of dyspnea in six patients with moderate to severe persistent bronchial asthma at the Department of Internal Medicine, Meiji University of Oriental Medicine, Japan. The combination of meridian points used for the treatment was Zhongfu, Chize, Tai Yuan, Guan Yuan, Zhong Wan, Fei Shu, Pishu, and Shen Shu [36]. Zhaohai and Lie Que are opening and coupling points for Ren Mai [37]. These points are used to maintain homeostasis of the lung and kidneys [38]. Although Zhaohai is not widely used for the treatment of asthma, and Lie Que is a connecting point for lung channel which is used widely in the treatment of asthma over the recent decades. It is an essential point in Chinese acupuncture according to the clinical trials for the treatment of asthma $[39,40]$. Although several local and distal acupoints including Back Shu are used to tonify and sedate heat and cold symptoms of lung in Sa-Ahm's acupuncture, the clinical use of such methods is still a concern. Yin meridians of water such as kidney meridian should be suppressed for maintaining a systemic balance [41]. Yin and yang meridians such as Shao Shang and Shang Yang are used to clear lung heat [42]. Fei Shu and acupoints of other meridians could be used to treat complications such as COPD [43].

These studies show that acupuncture could be used in the effective treatment of asthma through the stimulation of several meridian points.

4.3. Atherosclerosis. Electroacupuncture pretreatment was given at the bilateral Neiguan, Lieque, and Yunmen 
acupoints for 30 minutes each day for five consecutive days before surgery in sixty patients of the Fourth Military Medical University, Xi'an, China, suffering from heart valve disease. Limited troponin I release, reduction in ICU stay time, and decreased use of inotropic agents were the postoperative effects observed in the treated patients [44]. Thirty-five cases of The First Affiliated Hospital of Tianjin University of TCM, China, were randomized to receive acupuncture and moxibustion at certain acupoints including Renying, Neiguan, and $\mathrm{Zu}$ San $\mathrm{Li}$. The increase in size of the common carotid artery, reduction of intima-media thickness, accelerated peak systolic, and end-diastolic velocities, decreased pulsatility index, and resistance index were the effects observed after treatment [45]. 204 patients with coronary artery disease from 5 multicentered hospitals in China were registered for a clinical trial using electroacupuncture. All these patients under age 18 intended to receive percutaneous coronary intervention were treated with electroacupuncture for 30 minutes before the coronary intervention procedure. Stimulation at Neiguan and Ximen acupoints resulted in a decrease of the rate of major adverse cardiac/cerebrovascular events after a two-year follow-up [46].

Neiguan is the most used acupoint for the treatment of cardiac ailments by involving several mechanisms that improve angiogenesis and reduce apoptosis and calcium overload [47]. Zu San $\mathrm{Li}$ and auricular acupoints are other commonly used ones [48]. These studies indicate that acupuncture could be used in the improvement of blood flow and to alleviate the symptoms of atherosclerosis, thereby preventing their occurrence and development in vulnerable individuals.

4.4. Cancer. A case study was performed at Oregon College of Oriental Medicine, Oregon, USA, with a patient suffering from ductal carcinoma in situ. She received acupuncture treatment by stimulation at the Tai Chong (19 times), followed by $\mathrm{Zu}$ San $\mathrm{Li}$, He Gu, Qi Hai, Fenglong, and San Yin Jiao along with the use of four TCM extracts and a prescribed diet. This noninvasive form of breast carcinoma regressed by $75 \%$ over a treatment period of 15 months [49]. Zu San Li, Neiguan, Ge Shu, Fei Shu, Guan Yuan, and Shen Shu were the acupoints represented in their order of frequency of use in the treatment of lung cancer. A significant increase of IL-2, CD3+, and CD4+ T cells and NK cells were observed after the stimulation of these acupoints [50]. Electroacupuncture was clinically effective in the treatment of $55.6 \%$ of 36 patients with massive liver cancer at the ChinaJapan Friendship Hospital, Beijing, China [51]. IL-2 level and NK cell activities increased in peripheral blood of 25 patients with malignant tumors after acupuncture stimulation of 30 minutes for 10 days at acupoints Zu San Li, Quchi, and Qi Hai [52]. These studies determine that acupuncture could be effective in the treatment of patients with cancers of various origins.

4.5. Rheumatoid Arthritis. Thirty-six rheumatoid arthritis patients (mean age- $58 \pm 10$ years; disease duration-9.3 \pm 6.4 years) of the Prince of Wales Hospital were recruited for a randomized, double-blind, placebo-controlled trial at The
Institute of Chinese Medicine at The Chinese University of Hong Kong. All the patients received electroacupuncture and acupuncture for 20 sessions of two 40 minute-sessions every week over a 10-week period. Quchi, Waiguan, He Gu, $\mathrm{Zu}$ San Li, Yang Ling Quan, and Xuan Zhong were the acupoints used for the study. The pain score reduced in the treatment groups [17]. Laser acupuncture was employed at Tai Chong, Tianshu, Zu San Li, Houxi, Wan Gu, He Gu, Qu Chi, San Yin Jiao, Ying Ling Quan, Jing Men, Yang Ling Quan, and Shen Men acupoints of 15 arthritis patients aged 60-70 at the outpatient clinic of Kasr El Ainy Medical School and National Institute of Laser Enhanced Sciences, Cairo University, Egypt. Interleukin-6 and malondialdehyde levels decreased, whereas ATP levels and pain-free range of motion at joints increased [53]. Laser acupuncture (3 days/week for 4 weeks) was performed at $\mathrm{He} \mathrm{Gu,} \mathrm{Waiguan,} \mathrm{Qu} \mathrm{Chi,} \mathrm{Da} \mathrm{Zhui,}$ Tai Chong, San Yin Jiao, Yang Ling Quan, and Zu San Li acupoints at the National Research Centre (NRC) in Cairo, Egypt. After the treatment, increased plasma superoxide dismutase, glutathione reductase, catalase activities, blood glutathione, and plasma ATP concentrations were observed. Whereas plasma malondialdehyde, serum nitrate and nitrite, serum C-reactive protein, and interleukin-6 levels decreased. A Significant reduction in disease activity was observed based on the DAS28 score [54]. Distal points can be used to treat syndromes that could obstruct Qi, such as $\mathrm{Bi}$ syndrome, and needling at local points is applied for adjacent joints [17]. The abovementioned studies elucidated that laser acupuncture, electroacupuncture, and traditional acupuncture were effective in alleviating arthritis-related symptoms.

4.6. Stress and Anxiety. Stress can trigger inflammatory responses [55]. It can cause negative outcomes in affected individuals and is usually difficult to treat. A randomized controlled clinical trial with acupuncture decreased the perception of self-identified stress in affected college students, faculty, and staff at the Arizona State University, USA. The acupoints used were Bai Hui, Shen Men, Neiguan, Yintang, Four Gates, Dan Zhong, Qi Hai, and Zu San Li [56]. Heart rate variability increased in 10 patients, aged 33-72 who took acupuncture for hypertension in San Francisco, USA. A decrease in low-frequency-to-high-frequency $(\mathrm{LF} / \mathrm{HF})$ ratio of heart rate variability could be correlated to a decrease in physiological stress among the patients. The acupoints used were Zu San Li, Shang Ju Xu, Quchi, Shou San Li, Neiguan, and Da Ling [57]. Elevated inflammation is associated with anxiety [58]. A single acupuncture treatment at bilateral acupuncture point Shen Men was performed in twenty-five male subjects (aged $28 \pm 5$ years) at the University of Regensburg, Germany. Saliva samples were examined for cortisol and amylase. Anxiety questionnaires and heart rate variability were analyzed. The results indicate that there was a reduction in stress hormones and heart rate compared to existing reference data. Changes in other parameters were not significant [59]. Both auricular acupuncture and placebo interventions reduced the exam anxiety in forty-four medical students of University Medicine of Greifswald, Germany. The acupoints used were MA-IC1 (Lung), MA-TF1 (ear Shenmen), MA-SC (Kidney), MA-AT1 (Subcortex), and MA-TG (Adrenal 
TABLE 1: Location of the acupoints used in the treatment of chronic inflammation-oriented disorders.

\begin{tabular}{|c|c|c|c|}
\hline S. No. & Acupoint & Abbreviation & Location \\
\hline 1 & Bai Hui & GC 20 & 5 cun posterior to the anterior hairline \\
\hline 2 & Chize & LU 5 & $\begin{array}{l}\text { At the cubital crease on the radial side of } \\
\text { the biceps brachii tendon }\end{array}$ \\
\hline 3 & Da Ling & PC 7 & $\begin{array}{c}\text { In the middle of the wrist crease between the tendons of palmaris } \\
\text { ongus and flexor carpi radialis }\end{array}$ \\
\hline 4 & Da Zhui & DU 14 & $\begin{array}{l}\text { On the midline of the base of the neck, in the depression below the } \\
\text { spinous process of the 7th cervical vertebra }\end{array}$ \\
\hline 5 & Dan Zhong & CV 17 & Level with the 4 th intercostal space, midway between the nipples \\
\hline 6 & Fei Shu & BL 13 & $\begin{array}{c}1.5 \text { cun lateral to the lower border of the spinous process of the } \\
\text { third thoracic vertebra (T3) }\end{array}$ \\
\hline 7 & Fengchi & GB 20 & $\begin{array}{l}\text { In the depression created between the origins of the sternocleidomastoid } \\
\text { and trapezius muscles, at the junction of the occipital and nuchal regions }\end{array}$ \\
\hline 8 & Fenglong & ST 40 & $\begin{array}{l}\text { On the anterior of the leg midway between the crease of the knee } \\
\text { and the tip of the lateral malleolus } 2 \text { Cun lateral of the tibia }\end{array}$ \\
\hline 9 & Ge Shu & BL 17 & $\begin{array}{c}1.5 \text { cun lateral to the lower border of the spinous process } \\
\text { of the seventh thoracic vertebra (T7) }\end{array}$ \\
\hline 10 & Gong Sun & SP 4 & $\begin{array}{l}\text { On the medial aspect of the foot, in the depression distal } \\
\text { and inferior to the base of the first metatarsal bone }\end{array}$ \\
\hline 11 & Guan Yuan & CV 4 & $\begin{array}{l}\text { On the anterior midline of the lower abdomen, } \\
3 \text { cun below the umbilicus }\end{array}$ \\
\hline 12 & $\mathrm{He} \mathrm{Gu}$ & LI 4 & $\begin{array}{l}\text { On the dorsum of the hand, between the } 1 \text { st and } 2 \text { nd metacarpal bones, } \\
\text { in the middle of the } 2 \text { nd metacarpal bone on the radial side }\end{array}$ \\
\hline 13 & Hou Xi & SI 3 & $\begin{array}{c}\text { When a loose fist is made, the point is on the ulnar aspect } \\
\text { of the hand, proximal to the 5th metacarpophalangeal joint, } \\
\text { at the end of the transverse crease of the metacarpophalangeal joint, } \\
\text { at the junction of the red and white skin }\end{array}$ \\
\hline 14 & Jing Men & GB 25 & $\begin{array}{c}\text { On the lateral side of the abdomen on the lower } \\
\text { border of the free end of the } 12 \text { th rib }\end{array}$ \\
\hline 15 & Lie Que & LU 7 & $\begin{array}{l}\text { On the radial margin of the forearm, superior to the styloid } \\
\text { process of the radius, } 1.5 \text { cun above the transverse crease of the wrist }\end{array}$ \\
\hline 16 & Neiguan & PC 6 & $\begin{array}{l}\text { Three finger breadths below the wrist on the inner } \\
\text { forearm in between the two tendons }\end{array}$ \\
\hline 17 & Pishu & BL 20 & $\begin{array}{l}1.5 \text { cun lateral to the posterior midline, on the level of the lower border } \\
\text { of the spinous process of the } 11 \text { th thoracic vertebra (T11) }\end{array}$ \\
\hline 18 & Qi Hai & CV 6 & On the midline, 1.5 cun inferior to the umbilicus \\
\hline 19 & Quchi & LI 11 & $\begin{array}{c}\text { When the elbow is flexed, the point is in the midpoint between } \\
\text { the lateral end of the transverse cubical crease and the } \\
\text { lateral epicondyle of the humerus }\end{array}$ \\
\hline 20 & Ququan & LV 8 & $\begin{array}{l}\text { On the medial aspect of the knee, when the knee is flexed, } \\
\text { the point is in the depression on the medial end of the transverse } \\
\text { popliteal crease, on the posterior border of the medial epicondyle of } \\
\text { the femur, on the anterior portion of the insertion of semitendinosus } \\
\text { and semimembranosus muscles }\end{array}$ \\
\hline 21 & Renying & ST 9 & $\begin{array}{l}\text { Level with the tip of the Adam's apple on the anterior border of the } \\
\text { sternocleidomastoideus muscle (where the pulse of the common } \\
\text { carotid artery is felt) }\end{array}$ \\
\hline 22 & San Yin Jiao & SP 6 & On the inside of your leg, just above your ankle \\
\hline 23 & Shang Ju Xu & ST 37 & $\begin{array}{c}3 \text { cun below } \mathrm{Zu} \text { San } \mathrm{Li} \text {, one finger breadth from the anterior } \\
\text { crest of the tibia, in muscle tibialis anterior }\end{array}$ \\
\hline 24 & Shang Xing & GV 23 & At the top of the head, 1 cun posterior to the midline of the anterior hairline \\
\hline 25 & Shang Yang & LI 1 & .1 cun posterior to the corner of the nail on the radial side of the index finger \\
\hline 26 & Shao Shang & LU 11 & .1 cun posterior to the thumb nail on the radial side \\
\hline
\end{tabular}


TABLE 1: Continued.

\begin{tabular}{|c|c|c|c|}
\hline S. No. & Acupoint & Abbreviation & Location \\
\hline 27 & Shen Men & HT 7 & $\begin{array}{l}\text { At the wrist crease, on the radial side of the flexor carpi ulnaris tendon, } \\
\text { between the ulna and the pisiform bones }\end{array}$ \\
\hline 28 & Shen Shu & BL 23 & $\begin{array}{l}1.5 \text { cun lateral to the posterior midline, on the level of the lower } \\
\text { border of the spinous process of the } 2 \text { nd lumbar vertebra (L2) }\end{array}$ \\
\hline 29 & Shou San Li & LI 10 & $\begin{array}{l}\text { On the outer surface of the forearm and three fingers breadth } \\
\text { below the elbow crease when the elbow is bent } 90 \text { degrees }\end{array}$ \\
\hline 30 & Sibai & ST 2 & $\begin{array}{l}\text { With the eyes looking straight ahead, directly below the centre } \\
\text { of the pupil, in the depression at the infraorbital foramen }\end{array}$ \\
\hline 31 & Tai Chong & LV 3 & $\begin{array}{l}\text { On the dorsum of the foot, in a depression distal to } \\
\text { the junctions of the first and second metatarsal bones }\end{array}$ \\
\hline 32 & Tai Xi & KI 3 & $\begin{array}{l}\text { On the medial aspect of the foot, posterior to the medial malleolus, } \\
\text { in the depression between the tip of the medial malleolus } \\
\text { and tendo calcaneus }\end{array}$ \\
\hline 33 & Tai Yuan & LU 9 & At the wrist crease on the radial side of the radial artery \\
\hline 34 & Tian Shu & ST 25 & On the middle of the abdomen, 2 cun lateral to the umbilicus \\
\hline 35 & Waiguan & TE 5 & $\begin{array}{c}2 \text { cun proximal to the dorsal wrist crease between the radius and ulna, } \\
\text { close to the radial bone }\end{array}$ \\
\hline 36 & Wan Gu & SI 4 & $\begin{array}{l}\text { On the ulnar aspect of the palm, in the depression } \\
\text { between the 5th metacarpal bone and hamate bone, at the } \\
\text { junction of the red and white skin }\end{array}$ \\
\hline 37 & Xiaguan & ST 7 & $\begin{array}{l}\text { On the face, anterior to the ear, in a depression between the zygomatic } \\
\text { arch and the mandibular notch, with mouth closed }\end{array}$ \\
\hline 38 & Ximen & PC 4 & $\begin{array}{c}5 \text { cun above the wrist crease between the tendons of palmaris } \\
\text { longus and flexor carpi radialis }\end{array}$ \\
\hline 39 & Xuan Zhong & GB 39 & $\begin{array}{l}\text { On the lateral aspect of the lower leg, } 3 \text { cun above the tip of } \\
\text { the external malleolus, on the anterior border of the fibula }\end{array}$ \\
\hline 40 & Xue Hai & SP 10 & $\begin{array}{l}\text { When the knee is flexed, on the medial aspect of the thigh, } \\
\text { the point is } 2 \text { cun above the mediosuperior border of the patella, } \\
\text { on the bulge of the medial portion of m. quadriceps femoris }\end{array}$ \\
\hline 41 & Yang Ling Quan & GB 34 & In the depression anterior and inferior to the small head of the fibula \\
\hline 42 & Ying Ling Quan & SP 9 & $\begin{array}{l}\text { On the lower border of the medial condyle of the tibia in the } \\
\text { depression posterior and inferior to the medial condyle of the tibia }\end{array}$ \\
\hline 43 & Ying Xiang & LI 20 & $\begin{array}{c}\text { In the nasolabial groove, level with the midpoint of the } \\
\text { lateral border of the ala nasi }\end{array}$ \\
\hline 44 & Yintang & EX-HN3 & $\begin{array}{l}\text { At the forehead, at the midpoint between the } \\
\text { two medial ends of the eyebrow }\end{array}$ \\
\hline 45 & Yuji & LU 10 & Midpoint of the palmar border of the 1st metacarpal bone \\
\hline 46 & Yunmen & LU 2 & $\begin{array}{l}\text { On the latero-superior aspect of the chest, superior to the } \\
\text { coracoid process of scapula, in the depression of the } \\
\text { infraclavicular fossa, } 6 \text { cun lateral to the anterior median line }\end{array}$ \\
\hline 47 & Zhaohai & KD 6 & In a depression below the tip of the medial malleolus \\
\hline 48 & Zhong Wan & CV 12 & On the midline, 4 cun superior to the umbilicus \\
\hline 49 & Zhongfu & LU 1 & 6 cun lateral to the anterior midline, level with the 1 st intercostal space \\
\hline 50 & $\mathrm{Zu}$ san $\mathrm{Li}$ & ST 36 & Below the knee, on the tibialis anterior muscle, along the stomach meridian \\
\hline
\end{tabular}

Note: one cun is equal to the space between the distal interphalangeal joint and the proximal interphalangeal joint on the middle finger. Information of acupoint locations were obtained from the following websites: https://tcmwiki.com/,https://tcmwiki.com/, https://theory.yinyanghouse.com/acupuncturepoints/, https:// exploreim.ucla.edu/, https://www.sacredlotus.com/go/acupuncture/, https://www.acatcm.com/, https://www.acufinder.com/Acupuncture+Points/, http:// acupunctureschoolonline.com/, Retrieved on 24.01.2020.

gland). Yet, auricular acupuncture was effective in reducing exam anxiety as observed by visual analogue scales STAI and VAS-100 [60]. These clinical trials indicate that acupuncture is a possible candidate for the treatment of anxiety and stress.
4.7. Crohn's Disease. Moxibustion and acupuncture regulated the ratio of Th17 and Treg cells in the intestinal mucosa of Ninety-two patients with Crohn's disease. Moxibustion was performed on the Tian Shu, Qi Hai, and Zhong Wan acupoints, whereas acupuncture was performed on the $\mathrm{Zu}$ San 
TABLE 2: Outcomes of acupuncture treatment in clinical trials for inflammation-associated disorders.

\begin{tabular}{|c|c|c|c|c|}
\hline Method & Meridians & Outcome & Country & Ref \\
\hline \multicolumn{5}{|l|}{ Allergic rhinitis } \\
\hline $\mathrm{AC}$ & Xiaguan & Improvement of nasal obstruction and sneezing & China & 11 \\
\hline $\mathrm{AC}$ & Ying Xiang, Yintang, and Fengchi & Improvement of nasal and nonnasal symptoms & Australia & 12 \\
\hline $\mathrm{AC}$ & $\begin{array}{l}\text { Ying Xiang, Shang Xing, Fengchi, } \\
\text { Quchi, Xue Hai, Fei Shu, Ge Shu, } \\
\text { and Pishu }\end{array}$ & $\begin{array}{l}\text { Effects of acupuncture treatment were similar to } \\
\text { that of oral cetirizine hydrochloride treatment }\end{array}$ & China & 13 \\
\hline $\mathrm{AC}$ & $\begin{array}{c}\text { He Gu, Ying Xiang, Sibai, } \\
\text { Zu San Li, Yintang, and Shang Xing }\end{array}$ & $\begin{array}{l}\text { Reduction in total nasal and nonnasal } \\
\text { symptom score }\end{array}$ & $\begin{array}{l}\text { South Korea and } \\
\text { China }\end{array}$ & 14 \\
\hline \multicolumn{5}{|l|}{ Asthma } \\
\hline $\mathrm{AC}$ & Yuji & $\begin{array}{l}\text { Acupuncture effects comparable to the } \\
\text { effects of } 200 \mu \mathrm{g} \text { salbutamol aerosol }\end{array}$ & China & 18 \\
\hline $\mathrm{AC}$ & $\begin{array}{c}\text { Zhongfu, Chize, Tai Yuan, Guan Yuan, } \\
\text { Zhong Wan, Fei Shu, Pishu, } \\
\text { and Shen Shu }\end{array}$ & $\begin{array}{l}\text { Improved the symptoms of asthma and } \\
\text { VAS of dyspnea }\end{array}$ & Japan & 20 \\
\hline \multicolumn{5}{|l|}{ Atherosclerosis } \\
\hline EA & Neiguan, Lie Que, and Yunmen & $\begin{array}{l}\text { Limited troponin I release, reduction in } \\
\text { ICU stay time, and decreased use of } \\
\text { inotropic agents }\end{array}$ & China & 21 \\
\hline $\mathrm{AC}$ and Moxi & Renying, Neiguan, and Zu San Li & $\begin{array}{l}\text { Increase in size of common carotid artery, } \\
\text { reduction of intima-media thickness, } \\
\text { accelerated peak systolic and end } \\
\text { diastolic velocities, decreased pulsatility } \\
\text { index, and resistance index }\end{array}$ & China & 22 \\
\hline EA & Neiguan and Ximen & $\begin{array}{l}\text { Decrease of the rate of major adverse } \\
\text { cardiac/cerebrovascular events } \\
\text { after a two-year follow-up }\end{array}$ & China & 23 \\
\hline \multicolumn{5}{|l|}{ Cancer } \\
\hline $\begin{array}{l}\text { AC with herbs of } \\
\text { TCM }\end{array}$ & $\begin{array}{l}\text { Tai Chong, Zu San Li, He Gu, Qi Hai, } \\
\text { Fenglong, and San Yin Jiao }\end{array}$ & $\begin{array}{l}\text { Regression of ductal carcinoma over a } \\
\text { period of } 15 \text { months }\end{array}$ & USA & 26 \\
\hline $\mathrm{AC}$ & $\begin{array}{l}\text { Zu San Li, Neiguan, Ge Shu, Fei Shu, } \\
\text { Guan Yuan, and Shen Shu }\end{array}$ & $\begin{array}{l}\text { Significant increase of IL-2, CD3+ and CD4+ } \\
\text { T cells, and NK cells were observed }\end{array}$ & China & 27 \\
\hline $\mathrm{AC}$ & Zu San Li, Quchi, and Qi Hai & Increase in IL-2 level and NK cell activities & China & 29 \\
\hline \multicolumn{5}{|l|}{ Rheumatoid arthritis } \\
\hline $\mathrm{EA}$ and $\mathrm{AC}$ & $\begin{array}{l}\text { Quchi, Waiguan, He Gu, Zu San Li, } \\
\text { Yang Ling Quan, and Xuan Zhong }\end{array}$ & Reduction of pain score & China & 30 \\
\hline LA & $\begin{array}{l}\text { Tai Chong, Tianshu, Zu San Li, Houxi, } \\
\text { Wan Gu, He Gu, Qu Chi, San Yin Jiao, } \\
\text { Ying Ling Quan, Jing Men, } \\
\text { Yang Ling Quan, and Shen Men }\end{array}$ & $\begin{array}{l}\text { Interleukin- } 6 \text { and malondialdehyde levels } \\
\text { decreased, whereas ATP levels and pain-free } \\
\text { range of motion at joints increased }\end{array}$ & Egypt & 31 \\
\hline LA & $\begin{array}{c}\text { He Gu, Waiguan, Qu Chi, } \\
\text { Da Zhui, Tai Chong, San Yin Jiao, } \\
\text { Yang Ling Quan, and Zu San Li }\end{array}$ & $\begin{array}{l}\text { Increase in plasma superoxide dismutase, } \\
\text { glutathione reductase, catalase activities, } \\
\text { blood glutathione and plasma ATP } \\
\text { concentrations. Plasma MDA, serum nitrate } \\
\text { and nitrite, serum CRP, interleukin-6 levels } \\
\text { decreased. Significant reduction in disease } \\
\text { activity was observed based on DAS28 score. }\end{array}$ & Egypt & 32 \\
\hline \multicolumn{5}{|l|}{ Stress and anxiety } \\
\hline $\mathrm{AC}$ & $\begin{array}{l}\text { Bai Hui, Shen Men, Neiguan, } \\
\text { Yintang, Four Gates, Dan Zhong, } \\
\text { Qi Hai, and Zu San Li }\end{array}$ & $\begin{array}{c}\text { Decrease in the perception of self-identified } \\
\text { stress }\end{array}$ & USA & 34 \\
\hline $\mathrm{AC}$ & $\begin{array}{l}\text { Zu San Li, Shang Ju Xu, Quchi, } \\
\text { Shou San Li, Neiguan, and Da Ling }\end{array}$ & $\begin{array}{l}\text { Decrease in low-frequency-to-high-frequency } \\
\text { (LF/HF) ratio of heart rate variability }\end{array}$ & USA & 35 \\
\hline $\mathrm{AC}$ & Shen Men & Reduction in stress hormones and heart rate & Germany & 37 \\
\hline
\end{tabular}


TABLe 2: Continued.

\begin{tabular}{|c|c|c|c|c|}
\hline Method & Meridians & Outcome & Country & Ref \\
\hline $\mathrm{AC}$ & $\begin{array}{c}\text { MA-IC1 (lung), MA-TF1 } \\
\text { (ear Shenmen), MA-SC (kidney), } \\
\text { MA-AT1 (subcortex), and MA-TG } \\
\text { (adrenal gland) }\end{array}$ & Reduced exam anxiety & Germany & 38 \\
\hline \multicolumn{5}{|l|}{ Crohn's disease } \\
\hline Moxi and AC & $\begin{array}{l}\text { Tian Shu, Qihai, Zhongwan, } \\
\text { Zu San Li, Shang Ju Xu, } \\
\text { San Yin Jiao, Tai Xi, } \\
\text { Gong Sun, and Tai Chong }\end{array}$ & Decrease in the ratio of Th17 and Treg cells & China & 39 \\
\hline AC & $\begin{array}{l}\text { Ququan, Quchi, Zhong Wan, Qi Hai, } \\
\text { Zu San Li, and San Yin Jiao }\end{array}$ & $\begin{array}{l}\text { Improvement of symptoms such as } \\
\text { chronic indigestion, reflux, abdominal pain, } \\
\text { and excessive diarrhea }\end{array}$ & China & 40 \\
\hline Moxi and AC & $\begin{array}{l}\text { Tian Shu, Qi Hai, Zhong Wan, } \\
\text { Zu San Li, Shang Ju Xu, Gong Sun, } \\
\text { San Yin Jiao, Tai Xi, and Tai Chong }\end{array}$ & $\begin{array}{l}\text { Increased hemoglobin levels. Decreased CRP } \\
\text { levels and Crohn's disease activity index }\end{array}$ & China & 41 \\
\hline
\end{tabular}

AC: acupuncture; EA: electroacupuncture; Moxi: moxibustion; LA: laser acupuncture.

Li, Shang Ju Xu, San Yin Jiao, Tai Xi, Gong Sun, and Tai Chong acupoints [61]. In a case report, a 53-year-old woman suffering from refractory Crohn's disease was reported for treatment with acupuncture. Ququan, Quchi, Zhong Wan, Qi Hai, Zu San Li, and San Yin Jiao were the acupoints used. The frequency of treatment was once per week for a total of 21 sessions. The improvement of symptoms such as chronic indigestion, reflux, abdominal pain, and excessive diarrhea were observed after acupuncture treatment [19]. Ninety-two patients with Crohn's disease were treated with moxibustion at Tian Shu, Qi Hai, and Zhong Wan acupoints. Zu San Li, Shang Ju Xu, Gong Sun, San Yin Jiao, Tai $\mathrm{Xi}$, and Tai Chong were used for acupuncture in various medical centers around Shanghai, China. The hemoglobin increased, whereas the C-reactive protein levels decreased after treatment. The Crohn's disease Activity Index also decreased after treatment [62]. Pure, indirect moxibustion is preferred for clinical treatment of Crohn's disease $[61,63]$. These studies determine that acupuncture and moxibustion can have significant therapeutic benefits in patients with Crohn's disease.

The locations of acupoints used for the treatment of inflammation-associated disorders are enlisted in Table 1. The outcomes of acupuncture intervention are enlisted in Table 2.

\section{Adverse Effects}

Acupuncture is considered to be safe and known to possess limited side effects compared to clinical drugs. Although considered safe, the adverse events of acupuncture include the worsening of existing symptoms, dermatitis, fainting, fatigue, bruising, pain, and bleeding at the needling site. Needle breakage may lead to injuries in the lung and spinal cord, local argyria, dizziness, nausea, ocular, and sleeping disorders. Cardiovascular side effects such as endocarditis and tamponade were observed in clinical trials. The pulmonary side effects are pneumothorax and asthma. The inter- vention could even turn fatal by infections such as hepatitis B [64-68].

\section{Needling and Duration of Intervention}

Needling is performed at specific points to maintain a balance between yin and yang which is ultimate for the circulation of Qi [69]. Needling for 30 minutes is therapeutically effective for acute injuries, whereas 60 minutes is optimum for chronic injuries [70]. The duration of treatment for allergic rhinitis is 4 to 8 weeks $[30,31]$. The duration for atherosclerosis was 4 to 10 weeks [47, 48]. For cancer and arthritis, the duration was 2 to 8 weeks and 4 weeks, respectively $[50,53,54]$. The duration of intervention for stress ranged between 2 to 12 weeks $[56,57]$. For Crohn's disease, the duration was between 12 to 21 weeks [19, 61, 62]. The predominant duration was 4 weeks for the treatment of chronic inflammation-related diseases using acupuncture as per these reports.

\section{Challenges and Future Directions}

The ambiguous use of terminologies associated with acupuncture points is a critical challenge in therapy. Standard randomized controlled trials that adhere to the STRICTA and CONSORT criteria should be conducted in the future to ensure the effectiveness of acupuncture on chronic inflammation-related disorders. Blinding of parties including the patient and the evaluator are necessary to avoid conscious and unconscious bias in the design and execution of such clinical trials. Needling response should be clearly illustrated along with the experience of the acupuncture practitioner. Rigorous analysis of factors such as the pattern of stimulation, mode and dose, frequency of sessions, and overall duration of the treatment should be performed. Depth of needling is critical. Research on Acupuncture depth still remains underexplored and must be considered mandatory for future research. Mechanistic studies pertaining to peripheral 
and clinical metrics for determination of the specific effect of acupuncture are the need of the hour. Antiinflammatory effects of acupuncture are recently analyzed with care and are under the spotlight as acupuncture can possibly modulate inflammation.

\section{Conclusion}

Acupuncture depends on intervention in several acupoints and 12 meridians to maintain the homeostasis of the body through the release of neurotransmitters. Several reports have been published recently on the positive effects of acupuncture with regard to inflammation-oriented disorders. These reports suggest that Neiguan and $\mathrm{Zu} \mathrm{San} \mathrm{Li}$ are common acupoints used for acupuncture intervention of chronic inflammatory diseases. Risk assessment is of paramount importance in the fair, reasonable, and timely use of acupuncture for such diseases. Standard randomized controlled trials that adhere to approved criteria for practice and study the mechanisms involved are therefore the need of the hour to analyze the factors involved and predict the exact mode of action. The current review suggests that acupuncture could be applied to treat inflammation-related disorders in an appropriate manner.

\section{Conflicts of Interest}

The author(s) declare(s) that there are no conflicts of interest.

\section{Authors' Contributions}

Hua Bai and Senlei Xu contributed equally to this work.

\section{Acknowledgments}

This work was supported by the National Natural Science Foundation of China (81704169), the Natural Science Foundation of Jiangsu Province (BK20171067), and the Project of National Clinical Research Base of Traditional Chinese Medicine in Jiangsu Province, China (JD2019SZXYB03).

\section{References}

[1] B. X. Jin, L. L. Jin, and G.-Y. Jin, "The anti-inflammatory effect of acupuncture and its significance in analgesia," World Journal of Acupuncture - Moxibustion, vol. 29, no. 1, pp. 1-6, 2019.

[2] M. W. C. Chan, X. Y. Wu, J. C. Y. Wu, S. Y. S. Wong, and V. C. H. Chung, "Safety of acupuncture: overview of systematic reviews," Scientific Reports, vol. 7, no. 1, p. 3369, 2017.

[3] C. Vincent, "The safety of acupuncture," BMJ, vol. 323, no. 7311, pp. 467-468, 2001.

[4] Y. Zhuang, J.-j. Xing, J. Li, B.-Y. Zeng, and F.-r. Liang, "History of acupuncture research," International Review of Neurobiology, vol. 111, 2013.

[5] D. P. Lu and G. P. Lu, "An historical review and perspective on the impact of acupuncture on U.S. medicine and society," Med Acupunct, vol. 25, no. 5, pp. 311-316, 2013.

[6] A. Salehi, M. Marzban, and M. H. Imanieh, "The Evaluation of Curative Effect of Acupuncture," Journal of Evidence-Based
Complementary \& Alternative Medicine, vol. 21, no. 3, pp. 202-214, 2015.

[7] S. Schroeder, G. Meyer-Hamme, J. Zhang, S. Epplée, T. Friedemann, and W. Hu, "An acupuncture research protocol developed from historical writings by mathematical reflections: a rational individualized acupoint selection method for immediate pain relief," Evidence-based Complementary and Alternative Medicine, vol. 2013, 16 pages, 2013.

[8] M. Biçer, "The clinical efficiency of acupuncture in preventing migraine attacks and its effect on serotonin levels," Türkiye Fiziksel Tip ve Rehabilitasyon Dergisi, vol. 63, no. 1, pp. 5965, 2017.

[9] N. Maurer, H. Nissel, M. Egerbacher, E. Gornik, P. Schuller, and H. Traxler, "Anatomical evidence of acupuncture meridians in the human extracellular matrix: results from a macroscopic and microscopic interdisciplinary multicentre study on human corpses," Evidence-based Complementary and Alternative Medicine, vol. 2019, 8 pages, 2019.

[10] F. J. Zijlstra, I. van den Berg-de Lange, F. J. P. M. Huygen, and J. Klein, "Anti-inflammatory actions of acupuncture," Mediators of Inflammation, vol. 12, no. 2, 69 pages, 2003.

[11] H. Bao, D. Si, L. Gao et al., "Acupuncture for the treatment of allergic rhinitis: a systematic review protocol," Medicine, vol. 97, no. 51, p. e13772, 2018.

[12] D. P. Sniezek and I. J. Siddiqui, "Acupuncture for treating anxiety and depression in women: a clinical systematic review," Med Acupunct, vol. 25, no. 3, pp. 164-172, 2013.

[13] S. L. T. Goyatá, C. C. V. Avelino, S. V. M. d. Santos, D. I. d. Souza Junior, M. D. S. L. Gurgel, and F. d. S. Terra, "Efeitos da acupuntura no tratamento da ansiedade: revisao integrativa," Revista Brasileira de Enfermagem, vol. 69, no. 3, pp. 602-609, 2016.

[14] M. Li, X. Zhang, H. Bao, C. Li, and P. Zhang, "Acupuncture for asthma," Medicine, vol. 96, no. 26, p. e7296, 2017.

[15] L. Shi, J. Fang, J. Zhao et al., "Comparison of the therapeutic effects of acupuncture at PC6 and ST36 for chronic myocardial ischemia," Evidence-based Complementary and Alternative Medicine, vol. 2017, 9 pages, 2017.

[16] W. Lu, E. Dean-Clower, A. Doherty-Gilman, and D. S. Rosenthal, "The Value of Acupuncture in Cancer Care," Hematology/Oncology Clinics of North America, vol. 22, no. 4, pp. 631-648, 2008.

[17] L.-S. Tam, P.-C. Leung, T. K. Li, L. Zhang, and E. K. Li, “Acupuncture in the treatment of rheumatoid arthritis: a doubleblind controlled pilot study," BMC complementary and alternative medicine, vol. 7, no. 1, 2007.

[18] C.-Y. Kwon, B. Lee, and S.-H. Kim, "Effectiveness and safety of ear acupuncture for trauma-related mental disorders after large-scale disasters: a PRISMA-compliant systematic review," Medicine, vol. 99, no. 8, p. e19342, 2020.

[19] H. Lai, K. Wang, Q. Dong, X. Zhu, X. Li, and S. Qi, “Traditional Chinese medicine for management of recurrent and refractory Crohn disease: a case report," Medicine (Baltimore), vol. 98, no. 15, p. e15148, 2019.

[20] C.-H. Yeh, T. Y. Zhao, M. D. Zhao et al., "Comparison of effectiveness between warm acupuncture with local-distal points combination and local distribution points combination in breast cancer-related lymphedema patients: a study protocol for a multicenter, randomized, controlled clinical trial," Trials, vol. 20, no. 1, p. 403, 2019. 
[21] H. Tan, S. Tumilty, C. Chapple et al., "Understanding acupoint sensitization: a narrative review on phenomena, potential mechanism, and clinical application," Evidence-Based Complementary and Alternative Medicine, vol. 2019, 9 pages, 2019.

[22] C. Yang, T. T. Lv, T. Y. Yu, S. Wong, M. Q. Lu, and Y. Z. Li, "Acupuncture at tiaokou (ST38) for shoulder adhesive capsulitis: what strengths does it have? A systematic review and meta-analysis of randomized controlled trials," EvidenceBased Complementary and Alternative Medicine, vol. 2018, 11 pages, 2018.

[23] T.-K. Lim, Y. Ma, F. Berger, and G. Litscher, "Acupuncture and neural mechanism in the management of low back pain-an update," Medicine, vol. 5, no. 3, p. 63, 2018.

[24] M. Sun, S. Tao, G. Geng et al., "Identification of the optimal points for the acupuncture treatment of neck pain in China: protocol for a multicenter, matched, case-control study," BMJ Open, vol. 9, no. 8, p. e029194, 2019.

[25] A. Y. Fan, H. Ouyang, X. Qian et al., "Discussions on realworld acupuncture treatments for chronic low-back pain in older adults," Journal of integrative medicine, vol. 17, no. 2, pp. 71-76, 2019.

[26] R. D. Tan, Tan's Strategy of Twelve Magical Points, Richard Tan, San Diego, California, USA, 2002.

[27] O. T. Chong, H. O. D. Critchley, A. W. Horne, R. Elton, E. Haraldsdottir, and M. Fallon, "The BMEA study: the impact of meridian balanced method electroacupuncture on women with chronic pelvic pain-a three-arm randomised controlled pilot study using a mixed-methods approach," BMJ Open, vol. 5, no. 11, article e008621, 2015.

[28] J. Mi, X. Chen, X. Lin et al., "Treatment of persistent allergic rhinitis via acupuncture at the sphenopalatine acupoint: a randomized controlled trial," Trials, vol. 19, no. 1, p. 28, 2018.

[29] C. C. Xue, R. English, J. J. Zhang, C. Da Costa, and C. G. Li, "Effect of acupuncture in the treatment of seasonal allergic rhinitis: a randomized controlled clinical trial," The American Journal of Chinese Medicine, vol. 30, no. 1, pp. 1-11, 2012.

[30] Y.-d. Chen, X.-q. Jin, M.-h. Yu, Y. Fang, and L.-q. Huang, "Acupuncture for moderate to severe allergic rhinitis: a nonrandomized controlled trial," Chinese Journal of Integrative Medicine, vol. 22, no. 7, pp. 518-524, 2016.

[31] S. M. Choi, J.-E. Park, S.-S. Li et al., "A multicenter, randomized, controlled trial testing the effects of acupuncture on allergic rhinitis," Allergy, vol. 68, no. 3, pp. 365-374, 2013.

[32] G. W. K. Wong, "How should we treat patients with mild asthma?," New England Journal of Medicine, vol. 380, no. 21, pp. 2064-2066, 2019.

[33] L.-M. Yin, Y. Wang, L. Fan et al., "Efficacy of acupuncture for chronic asthma: study protocol for a randomized controlled trial," Trials, vol. 16, no. 1, 2015.

[34] J. Han, "Observation on effect of acupuncture at Yuji (LU 10) on the pulmonary function of patients with bronchial asthma and immediate efficacy of relieving asthma," Zhongguo zhen jiu = Chinese acupuncture \& moxibustion, vol. 32, pp. 891894, 2012.

[35] Z. L. Zhang, X. Q. Ji, L. Xue, and S. H. Yu, "Clinical observation on acupuncture for treatment of bronchial asthma at acute stage," Zhongguo zhen jiu = Chinese acupuncture \& moxibustion, vol. 25, pp. 158-160, 2005.

[36] M. Suzuki, K. Namura, M. Egawa, and T. Yano, "Effect of acupuncture treatment in patients with bronchial asthma," The
Journal of The Japan Acupuncture \& Moxibustion Society, vol. 56, pp. 616-627, 2006.

[37] M. T. Greenwood, "Non-duality, simplicity and the Chong Mai," Med Acupunct, vol. 30, no. 1, pp. 8-14, 2018.

[38] P. Sudhakaran and Y. M. Garcia, "How do you TreatAsthmain your practice?," Medical Acupuncture, vol. 30, no. 2, pp. 100112,2018

[39] M. Zhu, A handbook for treatment of acute syndromes by using acupuncture and moxibustion, 8 dragon publishing house, Hong Kong, 1991.

[40] K. A. Jobst, "Acupuncture in asthma and pulmonary disease: an analysis of efficacy and safety," The Journal of Alternative and Complementary Medicine, vol. 2, no. 1, pp. 179-206, 1996.

[41] C.-B. Ahn, K.-J. Jang, H.-M. Yoon et al., "A study of the SaAhm five element acupuncture theory," Journal of Acupuncture and Meridian Studies, vol. 2, no. 4, pp. 309-320, 2009.

[42] T. Z. Hong, "A close look at the application of the Yin-Yangbased acupoint pairs," Advancements in Bioequivalence o Bioavailability, vol. 2, 2019.

[43] F. Xie, Y. P. Wu, L. Liu, J. G. Ren, and B. D. Zhang, "Warm acupuncture on chronic obstructive pulmonary disease with phlegm-turbid obstructing of the lung: a randomized controlled trial," Zhongguo zhen jiu = Chinese acupuncture of moxibustion, vol. 39, pp. 918-922, 2019.

[44] L. Yang, J. Yang, Q. Wang et al., "Cardioprotective effects of electroacupuncture pretreatment on patients undergoing heart valve replacement surgery: a randomized controlled trial," The Annals of Thoracic Surgery, vol. 89, no. 3, pp. 781-786, 2010.

[45] W. L. Zhang and W. Z. Wang, "Impacts on carotid arterial vessel and blood flow in treatment of carotid atherosclerosis with acupuncture and moxibustion," Zhongguo zhen jiu = Chinese acupuncture \& moxibustion, vol. 31, pp. 783-785, 2011.

[46] Q. Wang, D. Liang, F. Wang et al., "Efficacy of electroacupuncture pretreatment for myocardial injury in patients undergoing percutaneous coronary intervention: a randomized clinical trial with a 2-year follow-up," International Journal of Cardiology, vol. 194, pp. 28-35, 2015.

[47] N. Wang, S.-F. Lu, H. Chen et al., "A protocol of histone modification-based mechanistic study of acupuncture in patients with stable angina pectoris," BMC Complementary and Alternative Medicine, vol. 15, no. 1, 2015.

[48] R. de Lima Pimentel, A. P. Duque, B. R. Moreira, and L. F. Rodrigues Junior, "Acupuncture for the treatment of cardiovascular diseases: a systematic review," Journal of Acupuncture and Meridian Studies, vol. 12, no. 2, pp. 43-51, 2019.

[49] R. Dehen, "Regression of ductal carcinoma in situ after treatment with acupuncture," The Journal of Alternative and Complementary Medicine, vol. 19, no. 11, pp. 911-915, 2013.

[50] H.-Y. Chen, S.-G. Li, W. C. S. Cho, and Z.-J. Zhang, "The role of acupoint stimulation as an adjunct therapy for lung cancer: a systematic review and meta-analysis," BMC Complementary and Alternative Medicine, vol. 13, no. 1, 2013.

[51] Y. Xin, D. Liu, and X. Meng, "Clinical effectiveness of electroacupuncture therapy in combination with invasive therapy for massive liver cancer," Zhonghua wai ke za zhi [Chinese journal of surgery], vol. 39, no. 10, pp. 756-758, 2001.

[52] B. Wu, R. X. Zhou, and M. S. Zhou, "Effect of acupuncture on interleukin-2 level and NK cell immunoactivity of peripheral blood of malignant tumor patients," Zhongguo Zhong xi yi jie he za zhi Zhongguo Zhongxiyi jiehe zazhi = Chinese journal 
of integrated traditional and Western medicine, vol. 14, pp. 537-539, 1994.

[53] A. S. Adly, A. S. Adly, M. S. Adly, and Z. M. H. Serry, "Laser acupuncture versus reflexology therapy in elderly with rheumatoid arthritis," Lasers in Medical Science, vol. 32, no. 5, pp. 1097-1103, 2017.

[54] A. M. M. Attia, F. A. A. Ibrahim, N. A. Abd El-Latif et al., "Therapeutic antioxidant and anti-inflammatory effects of laser acupuncture on patients with rheumatoid arthritis," Lasers in Surgery and Medicine, vol. 48, no. 5, pp. 490-497, 2016.

[55] V. Maydych, "The interplay between stress, inflammation, and emotional attention: relevance for depression," Frontiers in Neuroscience, vol. 13, 2019.

[56] S. Schroeder, J. Burnis, A. Denton, A. Krasnow, T. S. Raghu, and K. Mathis, "Effectiveness of acupuncture therapy on stress in a large urban college population," Journal of Acupuncture and Meridian Studies, vol. 10, no. 3, pp. 165-170, 2017.

[57] K. Sparrow and B. Golianu, "Does acupuncture reduce stress over time? A clinical heart rate variability study in hypertensive patients," Med Acupunct, vol. 26, no. 5, pp. 286-294, 2014.

[58] N. Vogelzangs, A. T. F. Beekman, P. de Jonge, and B. W. J. H. Penninx, "Anxiety disorders and inflammation in a large adult cohort," Translational Psychiatry, vol. 3, no. 4, p. e249, 2013.

[59] J. Fleckenstein, P. Krüger, and K.-P. Ittner, "Effects of singlepoint acupuncture (HT7) in the prevention of test anxiety: results of a RCT," PLoS One, vol. 13, no. 8, article e0202659, 2018.

[60] C. Klausenitz, H. Hacker, T. Hesse et al., "Auricular acupuncture for exam anxiety in medical students-a randomized crossover investigation," PLoS One, vol. 11, no. 12, article e0168338, 2016.

[61] C. Zhao, C. Bao, J. Li et al., "Moxibustion and Acupuncture Ameliorate Crohn's Disease by Regulating the Balance between Th17 and Treg Cells in the Intestinal Mucosa," Evidence-Based Complementary and Alternative Medicine, vol. 2015, 11 pages, 2015.

[62] C.-H. Bao, J.-M. Zhao, H.-R. Liu et al., "Randomized controlled trial: moxibustion and acupuncture for the treatment of Crohn's disease," World journal of gastroenterology: WJG, vol. 20, no. 31, pp. 11000-11011, 2014.

[63] C.-h. Bao, J.-z. Zhang, L.-y. Wu, J. Li, X.-q. Zeng, and H.-r. Liu, "Effect of electroacupuncture and herbal cake-partitioned moxibustion on anxiety and depression in patients with crohn's disease in remission," Journal of Acupuncture and Tuina Science, vol. 14, no. 2, pp. 87-92, 2016.

[64] A. Chung, L. Bui, and E. Mills, "Adverse effects of acupuncture. Which are clinically significant?," Canadian Family Physician, vol. 49, pp. 985-989, 2003.

[65] X. Xiao, J. Zhang, Y. Jin, Y. Wang, and Q. Zhang, "Effectiveness and safety of acupuncture for perimenopausal depression: a systematic review and meta-analysis of randomized controlled trials," Evidence-based Complementary and Alternative Medicine, vol. 2020, 13 pages, 2020.

[66] S.-Y. Chen, F.-S. Yieh, W.-L. Liao, T.-C. Li, and C.-L. Hsieh, "Effect of Acupuncture on Intraocular Pressure in Glaucoma Patients: A Single- Blinded, Randomized, Controlled Trial," Evidence-Based Complementary and Alternative Medicine, vol. 2020, 8 pages, 2020.
[67] J. Hasegawa, N. Noguchi, J. Yamasaki et al., "Delayed cardiac tamponade and hemothorax induced by an acupuncture needle," Cardiology, vol. 78, no. 1, pp. 58-63, 1991.

[68] S. Xu, L. Wang, E. Cooper et al., "Adverse events of acupuncture: a systematic review of case reports," Evidence-Based Complementary and Alternative Medicine, vol. 2013, 15 pages, 2013.

[69] J. D. Adams, "The effects of Yin, Yang and Qi in the skin on pain,” Medicines (Basel), vol. 3, no. 1, p. 5, 2016.

[70] L. U. Yong and J.-t. WANG, "An inquiry into the action of acupuncture on the human body by means of pharmacology," Journal of Traditional Chinese Medicine, vol. 29, no. 2, pp. 146-149, 2009. 